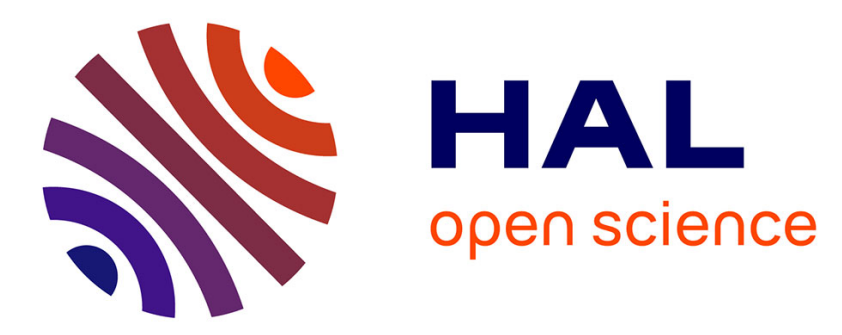

\title{
Unified approach of filament winding applied to complex shape mandrels
}

Erik Vargas, David Chapelle, Dominique Perreux, Benoit Delobelle, Frédéric

Thiebaud

\section{- To cite this version:}

Erik Vargas, David Chapelle, Dominique Perreux, Benoit Delobelle, Frédéric Thiebaud. Unified approach of filament winding applied to complex shape mandrels. Composite Structures, 2014, 116, pp.805-813. 10.1016/j.compstruct.2014.06.009 . hal-02300180

\section{HAL Id: hal-02300180 \\ https://hal.science/hal-02300180}

Submitted on 20 Nov 2019

HAL is a multi-disciplinary open access archive for the deposit and dissemination of scientific research documents, whether they are published or not. The documents may come from teaching and research institutions in France or abroad, or from public or private research centers.
L'archive ouverte pluridisciplinaire HAL, est destinée au dépôt et à la diffusion de documents scientifiques de niveau recherche, publiés ou non, émanant des établissements d'enseignement et de recherche français ou étrangers, des laboratoires publics ou privés. 


\title{
Unified approach of filament winding applied to complex shape mandrels
}

\author{
E. Vargas Rojas ${ }^{a}$, D. Chapelle ${ }^{a, *}$, D. Perreux ${ }^{a}$, B. Delobelle ${ }^{b}$, F. Thiebaud ${ }^{a}$ \\ ${ }^{a}$ Franche-Comté University, FEMTO-ST Institute, Department of Applied Mechanics, 24 rue de l'Épitaphe, 25000 Besançon, France \\ ${ }^{\mathrm{b}}$ MAHYTEC SARL, 210 Avenue de Verdun, 39100 Dole, France
}

\begin{abstract}
The filament winding process faces up limiting fabrication inconveniences when designing complex geometries of composite structures. Even the complete coverage of a cylindrical mandrel requires introducing deviation from geodesic trajectories. As a consequence, models for non-geodesic paths have been developed. The present research aims to establish, to solve and to validate a generic mathematical model that contributes either to wind complex shapes, or to solve common filament winding disadvantages, on the basis of an integrated strategy. This so-called unified approach leads to benefit of composite structures made by filament winding despite the limitations of the manufacturing process. Based on the mathematical description of the mandrel geometry, the theory of surfaces leads to express the local curvatures. Considering the slippage tendency of the fiber tow over the surface, a local stability criterion involving mathematical parameters of the mandrel surface is established, and a general fiber path equation can be formulated. A numerical tool is developed and applied to predict the evolution of the filament winding angle of the fiber tow placed over the surface of two axisymmetric geometries: a convex and a concave one. Experimental validation is carried out by manufacturing these geometries using a four axis filament winding machine.
\end{abstract}

\section{Introduction}

This research is motivated by the need to investigate the development of complex geometries in composite materials made by winding filament, i.e. non-cylindrical and non-symmetric objects. Certain industries such as automotive, aeronautical, or naval require specific solutions that go beyond the classical geometries such as cylinders, hemispheres or ellipsoids. In this respect, the HYPE [1] project (réservoir HYdrogène haute PrEssion - High pressure hydrogen reservoir), promoted by the French automotive industry, aimed to solve some of the drawbacks of hydrogen compressed storage technology for automotive applications like bulkiness, customer acceptance and cost, i.e. safety related issues to hydrogen behavior and high pressure. Considering these criteria, a new generation of reservoirs aims to take advantage of empty spaces in a car, to be as light as possible and not expensive. The main result was the development of form-fitted tanks as a geometrical evolution of type III (composite overwrapped metal liner) and IV (composite overwrapped polymer liner) tanks [2], see Fig. 1.

\footnotetext{
* Corresponding author. Tel.: +33 (0)381666029.

E-mail address: david.chapelle@univ-fcomte.fr (D. Chapelle).
}

Concerning other high performing solutions fabricated with the filament winding process, recent aeronautical solutions have constructed closed-shape structures [3,4], such as single-piece fuselages whose advantages include lower cost, lighter weight, improved integration, safety, improved performance, noise reduction, improved aerodynamics, and styling flexibility. For a onepiece fuselage, either metal or composite materials may be used. Metal has certain disadvantages due to the inability to fabricate all components of the fuselage in a single step; therefore composite materials are more advantageous for the fabrication of a one-piece fuselage because they can be fabricated simultaneously.

Filament winding structures have been achieved mostly using geodesic (friction-free) and non-geodesic paths. Geodesic winding paths are to be considered as natural fiber paths, i.e. the tension in the fiber tow causes the filament path to be geodesic regardless of the desired fiber path. The use of geodesic paths simplifies the calculation of fiber paths but is much more restrictive. The fiber trajectory is perfectly defined in so far as the initial position and the starting angle have been selected. Consequently, geodesic paths lead to an obstacle to optimization of the composite lay-up [5]. However, the filament path can be changed by altering the friction between the mandrel and the filament using some shrewdness 


\section{Nomenclature}

$\begin{array}{ll}F & \text { fiber tension force } \\ f_{b}, f_{f}, f_{n} & \text { lateral force, friction force, normal force } \\ k_{g}, k_{m}, k_{n}, k_{p} & \text { geodesic, meridian, normal, parallel curvatures } \\ L_{f} & \text { curve length (deposited fiber length); length of man- } \\ & \text { drel } \\ L_{c} & \text { length of concave and convex geometries } \\ L_{m} & \text { length of the mandrel } \\ \boldsymbol{P} & \text { point of analysis on } \boldsymbol{\Gamma} \text { curve } \\ \boldsymbol{M} & \text { centre of normal curvature } \\ \mathbf{N} & \text { centre of geodesic curvature } \\ \boldsymbol{n}_{u} \boldsymbol{n}_{v} & \text { derivatives in } u \text { - and } v \text {-direction of the unit normal } \\ \mathbf{O} & \text { vector to the surface } \\ R & \text { centre of curvature } \\ S & \text { cylindrical part radius } \\ \mathbf{S} & \text { length of the arc segment } \\ \mathbf{S}_{u}, \mathbf{S}_{v} & \text { surface; vector function describing the surface of the } \\ & \text { mandrel } \\ & \text { derivatives in } u \text { - and } v \text {-direction of the surface }\end{array}$

such as putting dressmaker pins, or applying high tack resin that keeps the filaments in place until a return crossing filament locks the previous ones as desired [4]. Other methods can be implemented such as using non-geodesic trajectories in which after following an initial rotation, no additional steps are taken to secure the tow of filaments at the desired angle [5-7].

But even the fabrication of simple geometries does not mean that the desired filament winding patterns are suitable, as demonstrated by [8]. In this case the geometrical parameters of a type III tank are dictated by the geometrical constraints of the liner rather than from a mechanical behavior calculation or optimization. Considering that the tank being analyzed by this author was a cylinder with ellipsoidal ends (or oblate spheroids) and taking into account mechanical strength of the structure, the desired winding angle of a helicoidally geodesic path over the cylindrical body should be $55^{\circ}$. Nevertheless, based on a sequential analysis of the filament winding process and considering a geodesic path, a $42^{\circ}$ angle is obtained on the cylindrical part as shown in Fig. $2 \mathrm{a}$; or $43.92^{\circ}$ angle when the path is non-geodesic as shown in Fig. 2 b. This is mainly due to the angle discontinuity of the transition between trajectories along the cylinder and the ellipsoids, as shown in Fig. 2c, so a non-geodesic transition should be considered. Furthermore, the dimension of the neck of the bottle imposes an entry angle (azimuth angle, $A_{z_{0}}$ ) of the fiber tow into the ellipsoids which also conditions the desired filament winding angle of the fiber placed over the cylinder. Therefore, in the previous analysis, the geometry of the supporting liner imposes the final geometrical description of the reinforcement.

$\begin{array}{ll}(u, v) & \begin{array}{l}\text { generalized curvilinear orthogonal coordinates; } \\ \text { parallel and meridian, respectively } \\ \text { derivative of fundamental coefficient } X \text { with respect } \\ \text { to } u \\ X_{u}\end{array} \\ \begin{array}{l}\text { machine rotating axis } \\ \text { curve orientation with respect to meridians (filament } \\ \text { winding angle) }\end{array} \\ (\varphi, \theta) \quad \begin{array}{l}\text { generalized curvilinear spherical-polar coordinates, } \\ \text { parallel and meridian, respectively }\end{array} \\ \boldsymbol{\Gamma} & \begin{array}{l}\text { curve; vector function describing a curve onto the } \\ \text { mandrel }\end{array} \\ \lambda & \begin{array}{l}\text { slippage tendency } \\ \text { static friction coefficient }\end{array} \\ \mu & \text { concave } \\ c c v & \text { convex } \\ c y x & \text { cylinder }\end{array}$

The scope of the present paper is to develop a numerical computing tool which will allow us to simulate the filament winding process whatever the mandrel geometry is, even if an axisymmetric mandrel is more suitable regarding the manufacturing process. By doing so, we contribute to the development of filament wound structures by designing, simulating and fabricating two axisymmetric geometries using a generic winding model that permits either to wind complex shapes, or to solve common filament winding disadvantages. To achieve this goal, a convex and a concave geometry are studied in an effort to propose more complex shapes, beyond traditional cylinders, or semi-spheres. Both shapes are described mathematically, so that the geodesic and the non-geodesic trajectories can be defined and solved. Then by means of a filament winding machine the previous complex geometries are roved and the numerical predictions are validated, allowing to corroborate the ability of the previous analysis to ensure that the referred mandrels can be overwrapped as desired. This work makes part of a more complete investigation which aims to define an optimization methodology: starting from the mathematical description of the mandrel, and knowing the mechanical loading, one should be able to suggest one or more suitable stacking sequences allowing to fulfill both objectives, a reduced weight and a high mechanical strength.

\section{Mathematical procedure}

The correct establishment of the stability condition of the fiber over the surface of the mandrel is the key to obtain the governing

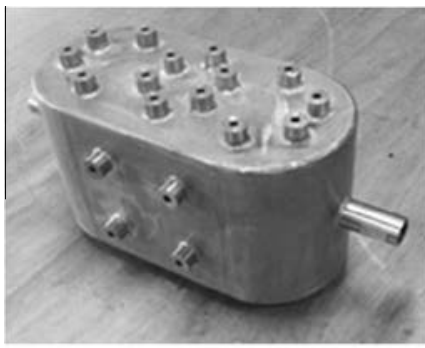

(a)

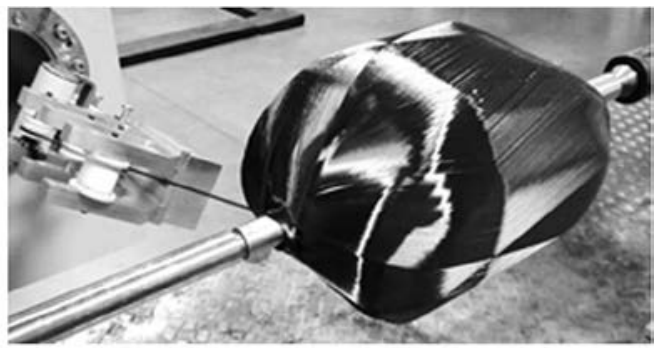

(b)

Fig. 1. Form-fitted tank (a) stainless steel liner. (b) Filament winding manufacturing [2]. 

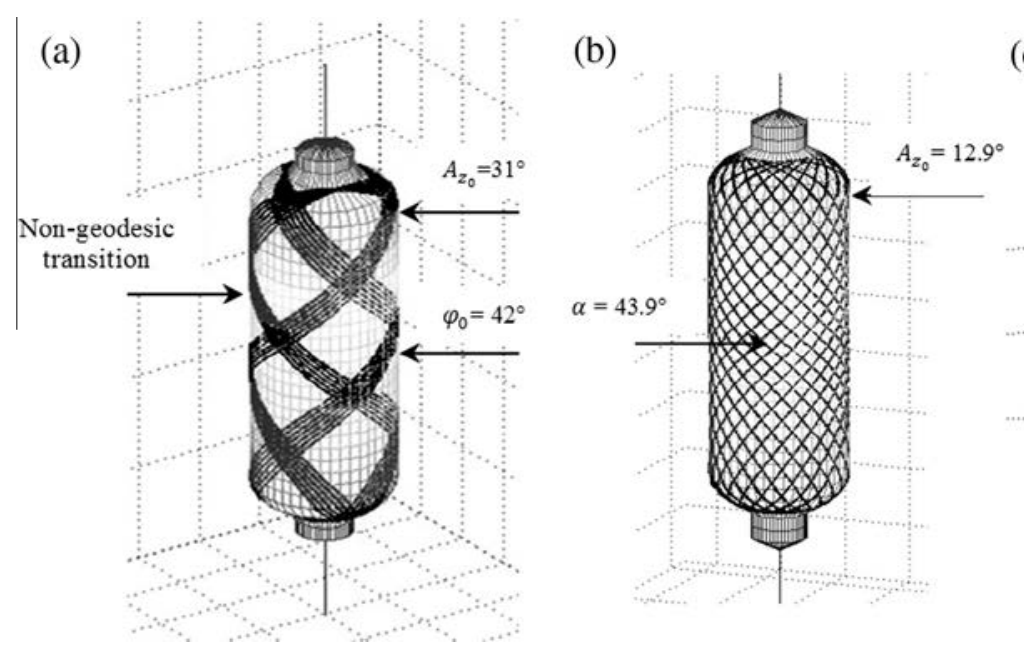

(c)

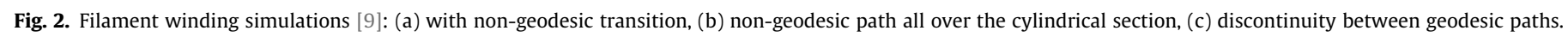

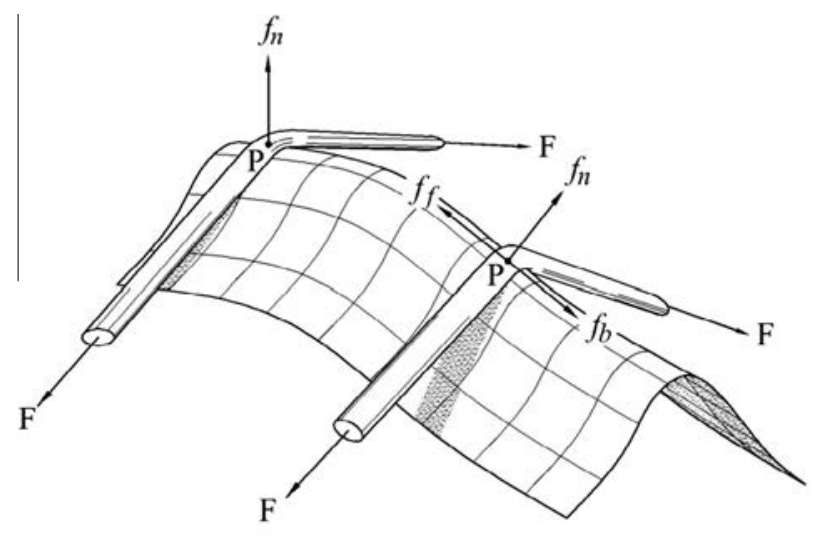

Fig. 3. Schematic representation of forces acting along a fiber path.

equation that defines the convenient curves over its surface. The stability of a trajectory depends on the ratio between the lateral force $f_{b}$, due to the change of the fiber angle (or due to the change of shape of the geometry); and the normal force $f_{n}$, which depends on the longitudinal force $F$ applied along the fiber, as illustrated in Fig. 3. It is assumed that the static coefficient of friction is $\mu$ and that $\lambda$ is a real parameter number whose values belong to the interval $[-\mu, \mu]$ called slippage tendency, defined as the ratio between the lateral force $f_{b}$ and the normal force $f_{n}$. No slipping of the fiber occurs when $\lambda<\mu$; or considering a Coulomb friction force $\left(f_{f}=\mu f_{n}\right)$, when $f_{b}$ is lower than the product of $\mu$ with $f_{n}$. As a direct consequence, and under the assumption of a local analysis, the previous relationship allows to describe a stable trajectory over the mandrel, condition that is defined by (1):

$$
\left|f_{b} / f_{n}\right| \leq \mu
$$

In a previous work [9], this same expression is derived in terms of the geodesic $k_{g}$ and the normal $k_{n}$ curvatures of the curve $\boldsymbol{\Gamma}$ traced over the surface $\mathbf{S}$ :

$\left|k_{g} / k_{n}\right|=|\lambda|$

The mathematical method presented here is based on the vector modeling of a surface $\mathbf{S}$ described by a mapping $\mathbf{S}(u, v)$ as a set of functions depending on two parameters, usually $\mathrm{u}$ and $\mathrm{v}$; and the parameterization of a curve in $\mathbb{R}^{3}$ represented as a function $\boldsymbol{\Gamma}$ to be placed over it, so as to conveniently define the geodesic and normal curvatures comprised in the stability criteria, expression (2). The theory of curves, as well as the theory of surfaces, plays a major role when considering this approach $[10,11]$.

In the free-body force diagram illustrated in Fig. 4a, a righthanded orthonormal triplet attached at each point $\mathbf{P}$ is formed by the unit tangent $\mathbf{t}$, the unit principal vector $\mathbf{p}$ (directed towards the centre of curvature), and the binormal $\mathbf{b}$ vector. The unit normal vector $\mathbf{n}$ could be considered as part of the referred orthonormal triplet instead of $\mathbf{p}$; but $\mathbf{n}$ arises certain uncertainty when defining its sense i.e. inwards or outwards the surface mapping at $\mathbf{P}$. The sense of $\mathbf{n}$ is important because certain criteria related with filament winding are based on its sense, like fiber bridging within mandrels with local concavities; according to De Carvalho [5] and Scholliers [12], it is defined considering $\mathbf{n}$ pointing outwards, so the product $\mathbf{n} f_{n} \leqslant 0$ (no fiber bridging occurs). Other criteria that depend on the sense of $\mathbf{n}$ is the sense of the curvature vector, considered positive when $\mathbf{n}$ points inwards the osculating circle tangent to $\mathbf{P}$ of a planar curve [13]. In this sense, Kreyszig [11] introduces the concept of normal vector to a surface rather than to a curve. In this document the criteria that has been used in filament winding literature is preferably adopted.

Normal curvature is the curvature of the curve of intersection (normal section) of the surface and a plane passing through both the tangent to the curve at $\mathbf{P}$ and the unit normal vector $\mathbf{n}$. Normal curvature vector $\mathbf{k}_{\boldsymbol{n}}$ is expressed as a vector projection (3) of curvature vector $\mathbf{k}$ onto the unit normal vector $\mathbf{n}$. Geodesic curvature vector $\mathbf{k}_{\mathbf{g}}$ is the vector projection (4) of curvature vector $\mathbf{k}$ onto the binormal vector b. Both curvature vectors, and its resultant, are illustrated in Fig. 4b.

$$
\begin{aligned}
& \mathbf{k}_{\boldsymbol{n}}=(\mathbf{k} \cdot \mathbf{n}) \mathbf{n}=k_{n} \mathbf{n} \\
& \mathbf{k}_{\boldsymbol{g}}=(\mathbf{k} \cdot \mathbf{b}) \mathbf{b}=k_{\mathrm{g}} \mathbf{n}
\end{aligned}
$$

There is an infinite number of planes passing through $\mathbf{P}$ containing the unit normal vector $\mathbf{n}$, i.e. an infinite number of normal sections passing through $\mathbf{P}$. Each of these normal sections possesses a particular curvature at $\mathbf{P}$. It can be shown that a maximum and a minimum value exist and that the directions of the tangent lines corresponding to these extreme curvatures are orthogonal (also known as principal directions). By drawing a polar coordinate system on the tangent plane with its pole at $\mathbf{P}$, and its polar axis at some arbitrarily chosen ray, an angle $\alpha$ measured counterclockwise from the polar axis allows to draw a function $k_{n}=f(\alpha)$. So, for each value of $\alpha$ there is a curvature associated with that particular 

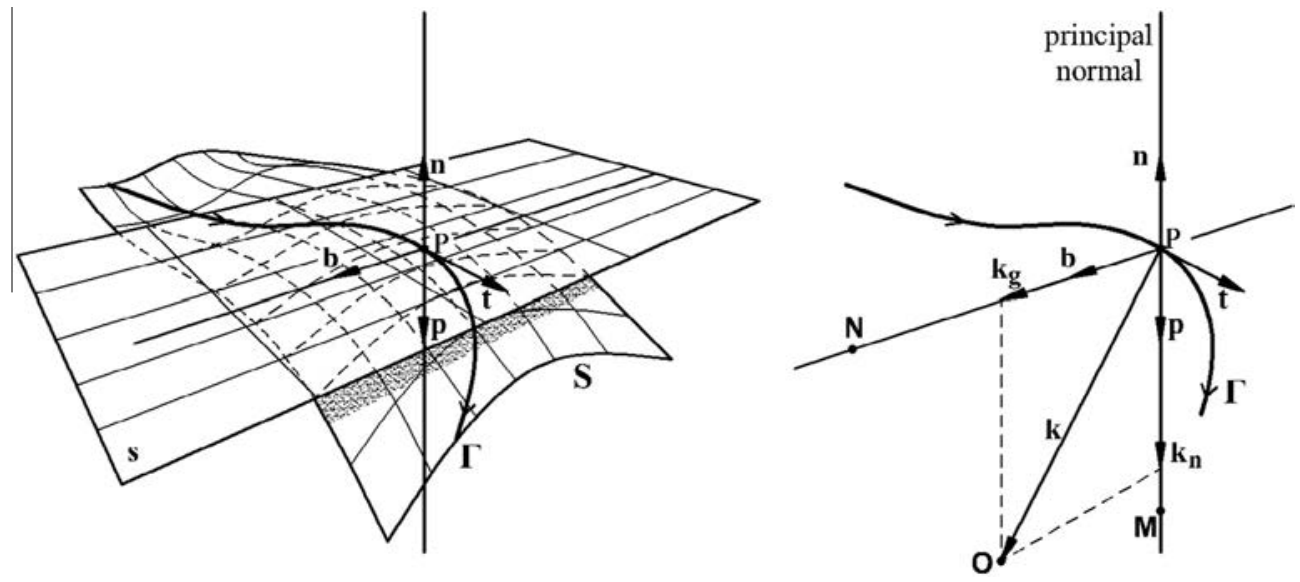

Fig. 4. Schematic illustrations: (a) curve and surface reference frames. (b) Curve related vectors.

normal section; this curvature $k_{n}$ is the normal curvature of surface $\mathbf{S}$ at point $\mathbf{P}$ in the direction $\alpha$ and it is given by (5), known as the Euler's theorem $[10,11]$ :

$k_{n}=k_{1} \cos ^{2}(\alpha)+k_{2} \sin ^{2}(\alpha)$

where $k_{1}$ and $k_{2}$ are the principal curvatures at $\mathbf{P}$ and $\alpha$ is the angle between the tangent line in the principal direction corresponding to $k_{1}$. Eq. (5) is an important expression that allows to introduce the term $\alpha$ as the orientation of the curve $\boldsymbol{\Gamma}$ with respect to meridians, i.e. the rotating axis. As a consequence, $\alpha$ is the filament winding angle. Now, if $s$ parameter, the length of the arc segment, is introduced to describe $\boldsymbol{\Gamma}: s \mapsto \mathbf{S}(u(s), v(s))$, i.e. the natural parameterization, the normal curvature can be expressed as a function of the first $(E, F, G)$ and second $(L, M, N)$ fundamental coefficients, which are continuous functions of $u$ and $v$; hence:

$k_{n}(s)=L \beta^{2}+2 M \beta \gamma+N \gamma^{2}$

where $\beta=d u / d s, \gamma=d v / d s, E=\mathbf{S}_{u} \cdot \mathbf{S}_{u}, F=\mathbf{S}_{u} \cdot \mathbf{S}_{u}, G=\mathbf{S}_{v} \cdot \mathbf{S}_{v}, L=\mathbf{n} \cdot \mathbf{S}_{u u}$, $M=\mathbf{n} \cdot \boldsymbol{S}_{u v}, N=\mathbf{n} \cdot \mathbf{S}_{v v}$. The directions in which the normal curvature at $\mathbf{P}$ reaches its maximum and minimum values are obtained by dividing $k_{n}(s)$ by $\beta^{2}$, then solving the equation $d k_{n}(\delta) / d \delta=0$ where $\delta=d v / d u$. So, after expanding and rearranging, a quadratic formula is obtained allowing to calculate the two principal directions $\delta_{1,2}$, or as stated in (5), $k_{1,2}$.

For a surface of revolution $\mathbf{S}$ the principal curvatures are given by (7) and (8) in terms of first and second fundamental coefficients [14]; $k_{1}$ corresponds to the meridian curvature $k_{m}$, and $k_{2}$ to the parallel one $k_{p}$.

$k_{1}=k_{m}=N / G$

$k_{2}=k_{p}=L / E$

Geodesic curvature depends only on the first fundamental coefficients and their derivatives, and is hence an intrinsic property of the surface $\mathbf{S}$. Then assuming a regular, continuous, and naturally represented curve $\boldsymbol{\Gamma}$ is considered, the geodesic curvature is given by (9):

$$
\begin{aligned}
k_{g}(s)= & \Gamma_{11}^{2}\left(\frac{d u}{d s}\right)^{3}+\left(2 \Gamma_{12}^{2}-\Gamma_{11}^{1}\right)\left(\frac{d u}{d s}\right)^{2}\left(\frac{d v}{d s}\right) \\
& +\left(\Gamma_{22}^{2}-2 \Gamma_{12}^{1}\right)\left(\frac{d u}{d s}\right)\left(\frac{d v}{d s}\right)^{2}-\Gamma_{22}^{1}\left(\frac{d v}{d s}\right)^{3}+\left(\frac{d u}{d s}\right)\left(\frac{d^{3} v}{d s^{3}}\right) \\
& \left.-\left(\frac{d v}{d s}\right)\left(\frac{d^{3} u}{d s^{3}}\right)\right) \sqrt{E G-F^{2}}
\end{aligned}
$$

where $\Gamma_{i j}^{k}$ are the Christoffel symbols of the second kind that are function of $E, F, G, E_{u}, E_{v}, F_{u}, F_{v}, G_{u}, G_{v}$. The geodesic curvature definition allows the integration of the well-known Clairaut relation [15], together with the evaluation of geodesic trajectories.

By substituting (7) and (8) onto (5), an expression for $k_{n}$ is obtained, and by re-substituting it as well as (9) onto (2), a general path equation for $\alpha=f(u, v)$ is derived. In order to obtain the desired expression that defines the general path equation for a surface of revolution it is required that $\mathbf{S}$ and $\boldsymbol{\Gamma}$ to be derivable, so that their geometrical parameters can be calculated [10]. From this statement $\mathbf{S}$ should be continuous and regular, defined as a function of meridian $\theta$ and parallel $\varphi$ coordinates defined on a spherical-polar frame. For an axisymmetric mandrel, $\mathbf{S}$ is described according to the following Eq. (10) whose origin is located on the revolution axis, being $g(\theta)$ the function that describes the profile.

$\mathbf{S}(\varphi, \theta)=\{g(\theta) \sin (\theta) \cos (\varphi), g(\theta) \sin (\varphi), g(\theta) \cos (\theta)\}$

Main curvatures, i.e. normal and parallel ones are obtained with (11) and (12), respectively:

$k_{m}(\theta)=\frac{N}{G}=\frac{2 \times\left(\frac{d g(\theta)}{d \theta}\right)^{2}-\mathrm{g}(\theta) \times \frac{d^{2} g(\theta)}{d \theta^{2}}+g^{2}(\theta)}{\left(g^{2}(\theta)+\left(\frac{d g(\theta)}{d \theta}\right)^{2}\right)^{3 / 2}}$

$k_{p}(\theta)=\frac{L}{E}=\frac{\mathrm{g}(\theta) \times \sin (\theta)-\frac{\mathrm{dg}(\theta)}{d \theta} \times \cos (\theta)}{\mathrm{g}(\theta) \times \sin (\theta) \times\left(\mathrm{g}^{2}(\theta)+\left(\frac{d \mathrm{~g}(\theta)}{d \theta}\right)^{2}\right)^{1 / 2}}$

The terms necessary to determine the geodesic curvature simplify considerably because for a surface of revolution $F=0$ and $M=0$. By substituting the respective Christoffel symbols of the second kind in terms of the first fundamental coefficients and their derivatives, the geodesic curvature (9) becomes as follows:

$k_{g}=-\frac{d \alpha(\theta)}{d \theta} \frac{\cos (\alpha(\theta))}{\sqrt{G(\theta)}}-\frac{1}{2} \frac{E_{\theta}}{E} \frac{\sin (\alpha(\theta))}{\sqrt{G(\theta)}}$

Geodesic curvature (13) and normal curvature (5) in terms of meridian (11) and parallel (12) curvatures are substituted in the stability condition criteria (2) to finally obtain a general path ordinary differential equation ODE for $\alpha$ as a function of meridian coordinate $\theta$ (14); the equations that define the parametric coupling of $\theta$ and $\varphi$, and the length $L_{f}$ of the trajectory, between points $\mathbf{P}\left(\theta_{a}\right)$ and $\mathbf{P}\left(\theta_{b}\right)$, are expressed with (15) and (16), respectively. 


$$
\begin{aligned}
\frac{d \alpha(\theta)}{d \theta}= & \frac{1}{2} \frac{E_{\theta}}{E} \tan (\alpha(\theta)) \\
& \left.+\lambda \frac{\sqrt{G}}{\cos (\alpha(\theta))}\left(k_{m} \cos ^{2}(\alpha(\theta))+k_{p} \sin ^{2}(\alpha(\theta))\right)\right)
\end{aligned}
$$

$\varphi(\theta)=\int_{\theta_{a}}^{\theta_{b}} \sqrt{\frac{G}{E}} \tan (\alpha(\theta)) d \theta$

$L_{f}(\theta)=\int_{\theta_{a}}^{\theta_{b}} \frac{\sqrt{G}}{\cos (\alpha(\theta))} d \theta$

The expression of $\varphi$ angle vs. $\theta$ angle leads to a complete description of the curve $\Gamma$ :

$\boldsymbol{\Gamma}(\theta)=\{g(\theta) \sin (\theta) \cos (\varphi(\theta)), g(\theta) \sin (\theta) \sin (\varphi(\theta)), g(\theta) \cos (\theta)\}$

\section{Resolution procedure}

The numerical method for solving and computer simulation modeling comprises various functions: input data, design of mandrel geometry, design of one fiber path, design of global coverage pattern, and calculation of the machine paths. Input data comprises parameters of the mandrel, plus fiber parameters. As indicated in the ODE (14), one fundamental parameter when designing fiber paths is the slipping tendency, strictly speaking the static coefficient of friction. According to [12], the value of $\mu$ depends on various factors, such as resin viscosity and mandrel surface finish, and can vary between 0.2 and 0.4 . Nowadays, higher friction coefficient values are regular when using thermosetting Prepregs. Here a 0.2 value for the static coefficient of friction is selected, i.e. the achievable deviation from the geodesic trajectory is minimized so as the slipping risk is simultaneously limited.

Aiming to prove the feasibility of the mathematical approach to simulate, but also to wind complex geometries, two case studies whose profiles are illustrated in Fig. 5 are proposed: a convex and a concave geometry.

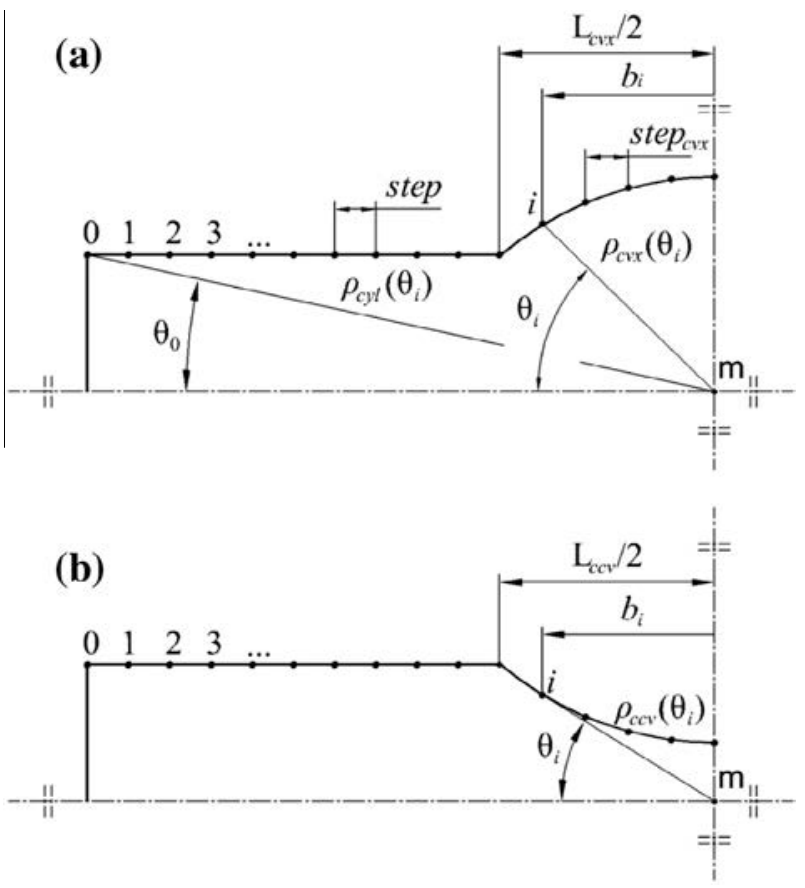

Fig. 5. Complex shape mandrel description: (a) convex-shaped profile, (b) concaveshaped profile.
The design of the mandrel geometry comprises the mathematical definition of $\rho=\rho(\theta)$; i.e. a discretized contour function. Considering that the center of each geometry is located at point $\mathrm{m}$, the $\rho(\theta)$ functions describing the cylinders, the convex shape and the concave shape contours of the mandrels are respectively given by Eqs. (18)-(20).

$\rho_{\text {cyl }}(\theta)=R / \sin (\theta)$

$\rho_{c v x}(\theta)=\sqrt{\mathrm{L}_{c}^{2}+3 R^{2}+R^{2} \sin ^{2}(\theta)}-R \sin (\theta)$

$\rho_{c c v}(\theta)=\sqrt{\mathrm{L}_{c}^{2}+4 R^{2}-9 R^{2} \cos ^{2}(\theta)}+3 R \sin (\theta)$

The continuity between the cylindrical and either the convex (19) or the concave (20) geometry is ensured with the product of sigmoid functions, one for the cylindrical part, $f_{1}$, and the other for the geometry under analysis, $f_{2}$. For the convex geometry mandrel, the contour is constructed with Eq. (21).

$$
\begin{aligned}
g(\theta)= & \left(1-\left(1+f_{1}\right)^{-1} \cdot\left(1+f_{2}\right)^{-1}\right) \rho_{c y l}(\theta) \\
& +\left(\left(1+f_{1}\right)^{-1} \cdot\left(1+f_{2}\right)^{-1}\right) \rho_{c v x}(\theta)
\end{aligned}
$$

where:

$f_{1}(\theta)=e^{-a\left(b+L_{c}\right)}, \quad f_{2}(\theta)=e^{-a\left(b-L_{c}\right)}, \quad b=\frac{R}{\tan (\theta)}$

In the previous equations, $a$ is the parameter that defines the transition spread and $b$ the transition position of the sigmoid function.

Considering its accuracy and stability, ODE (14) is solved by the modified Euler method, derived by applying the trapezoidal rule to a solution of type $\alpha_{\theta}=f(\alpha, \theta)$, Eq. (22), [16]. For this purpose, $\theta$ angle is discretized.

$\alpha_{i+1}=\alpha_{i}+h\left(f\left(\alpha_{i+1}, \theta_{i+1}\right)+f\left(\alpha_{i}, \theta_{i}\right)\right) / 2$

where $h$ is the step size, $\alpha$ is the independent coordinate, and $\theta$ the dependent coordinate. Initial condition is $\alpha\left(\theta_{0}\right) \approx \pi / 2$, i.e. the fiber is placed lightly - so as to prevent a numerical error - perpendicular to the generatrix lines at the beginning of the cylindrical part of the mandrel, what is commonly known as a hoop pattern.

Numerical differentiation required by Eqs. (11) and (12)is achieved using the centered finite-difference method using Eqs. (23) and (24)relative to $f$ function, [17]. The numerical integration of (15) and (16) is realized considering the trapezoidal rule (25) and (26).

$f_{\theta}\left(\theta_{i}\right)=\left(-f\left(\theta_{i+2}\right)+8 f\left(\theta_{i+1}\right)-8 f\left(\theta_{i-1}\right)+f\left(\theta_{i-2}\right)\right) / 12 h$

$f_{\theta \theta}\left(\theta_{i}\right)=\left(-f\left(\theta_{i+2}\right)+16 f\left(\theta_{i+1}\right)-30 f\left(\theta_{i}\right)+16 f\left(\theta_{i-1}\right)-f\left(\theta_{i-2}\right)\right) / 12 h^{2}$

$\varphi_{i}=\varphi_{i-1}+\frac{1}{2}\left(\theta_{i}-\theta_{i-1}\right)\left(\sqrt{G_{i} / E_{i}} \tan \left(\alpha_{i}\right)+\sqrt{G_{i-1} / E_{i-1}} \tan \left(\alpha_{i-1}\right)\right)$

$\mathrm{L}_{f_{i}}=\mathrm{L}_{f_{i-1}}+\frac{1}{2}\left(\theta_{i}-\theta_{i-1}\right)\left(\sqrt{G_{i}} / \cos \left(\alpha_{i}\right)+\sqrt{G_{i-1}} / \cos \left(\alpha_{i-1}\right)\right)$

The complete methodology is implemented with MATLAB ${ }^{\circledR}$ software; based on the mathematical description of the mandrels, it allows calculating the fiber path all along the mandrel length $z$ step by step. Figs. 6-8 illustrate the results that are obtained for three different mandrels; for each mandrel three plots are attached: the distribution of filament winding angle $\alpha$ as a function of $\theta$, the evolution of $\varphi$ along the length of the mandrel ( $z$ direction), and the length of the trajectory $L_{f}$ of the fiber with 
(a)

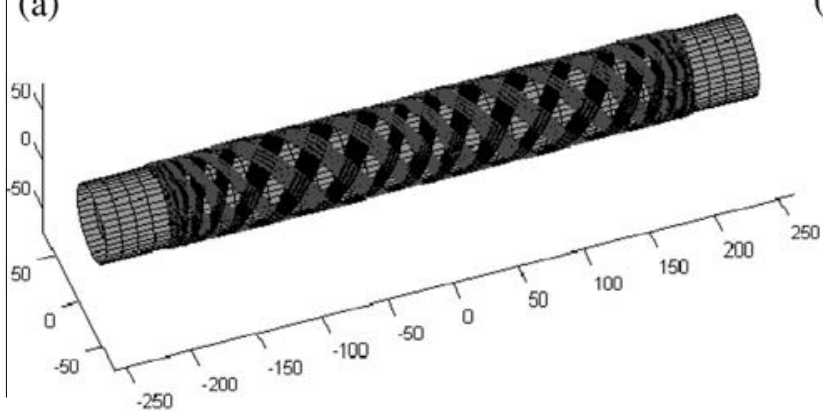

(b)

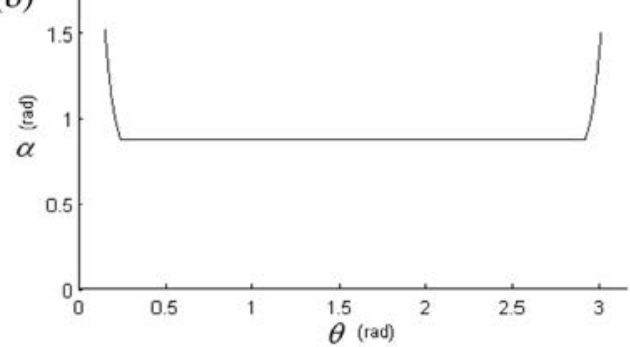

(c)

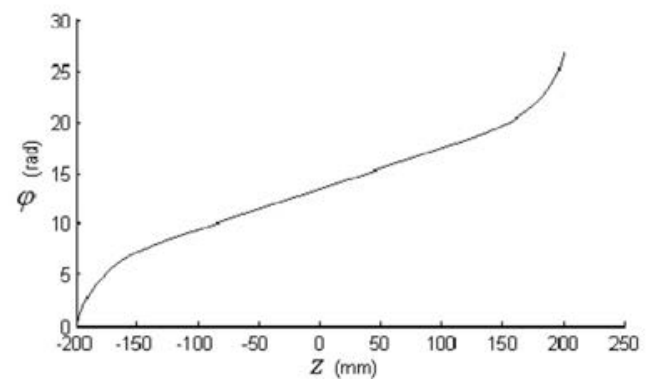

(d)

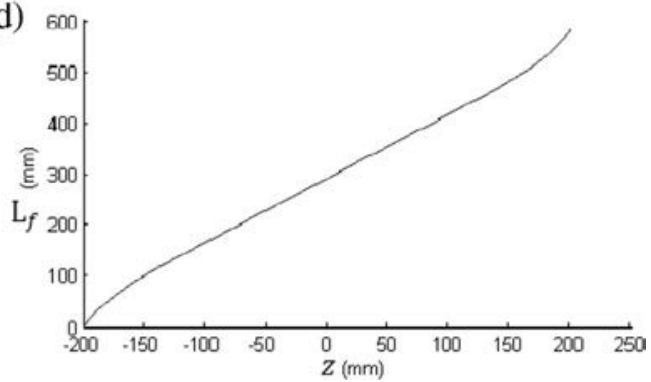

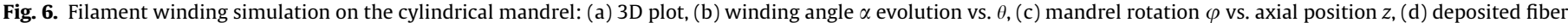
length $L_{f}$ vs. axial position $z$.

(a)

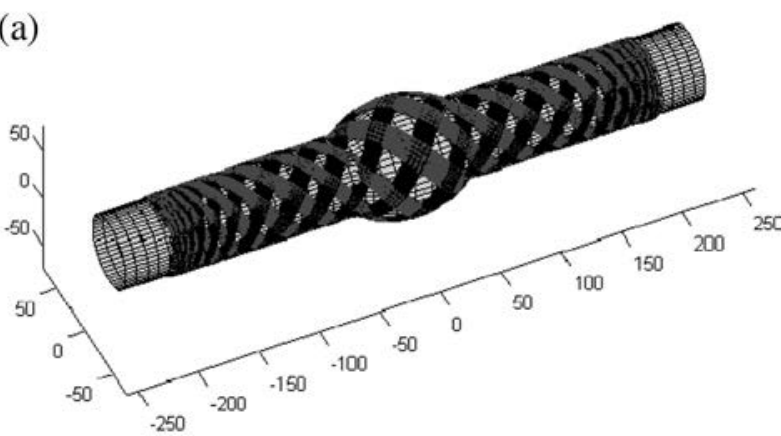

(c)

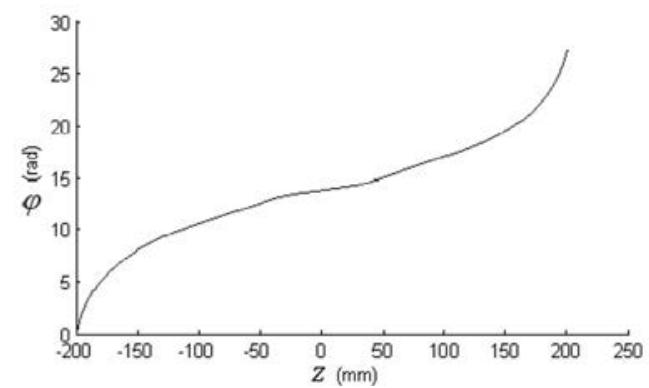

(b)

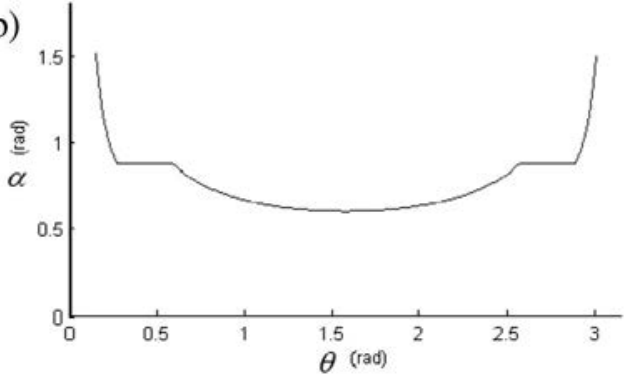

(d)

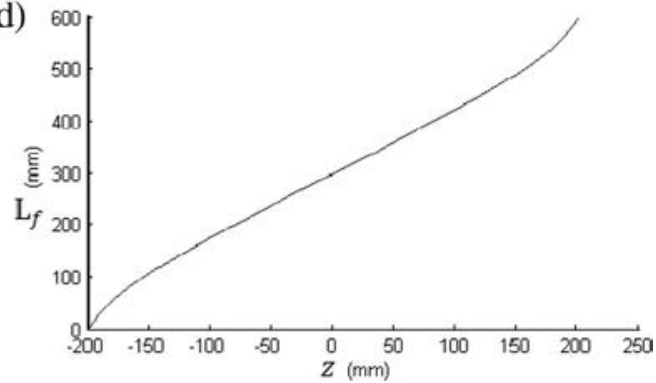

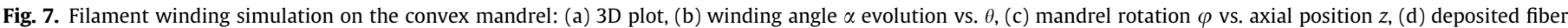
length $L_{f}$ vs. axial position $z$.

respect to $z$. The numerical resolution of ODE (14) has been compared and validated with its analytical solution obtained for a cylinder (27); where $\xi$ is the ratio of the mandrel mid-length with the cylinder radius; and $\omega$ is part of a relationship that allows to calculate the initial winding angle [9].

$\alpha=\arcsin \left((\omega+\lambda(\cot (\theta)-\xi))^{-1}\right), \quad$ where

$\xi=0.5 L_{m} / R$ and $\sin \left(\alpha_{\theta_{0}}\right)=1 / \omega$

The pattern to be over-wound may need to respond to mechanical solicitations, so it becomes necessary to define a strategy that ensures the full and uniform coverage of the surface. Several strategies $[8-9,19-23]$ that program a single circuit of the fiber wind path have been further developed, making it a repeatable band pattern, so the circuit can be countless repeated to provide one or numerous fiber layers completely covering the mandrel. In this section, we consider the monofilament winding technique proposed by $[8,23]$ that allows to control highly interwoven winding patterns.

Given any axisymmetric geometry at a certain circular section, the number of single circuits (the path followed from one extreme to the other without returning) required to cover its surface is determined by considering a fiber band of true width $\mathrm{w}_{\mathrm{t}}$, and the winding angle $\alpha$, thus the width of the band along the hoop direc- 
(a)

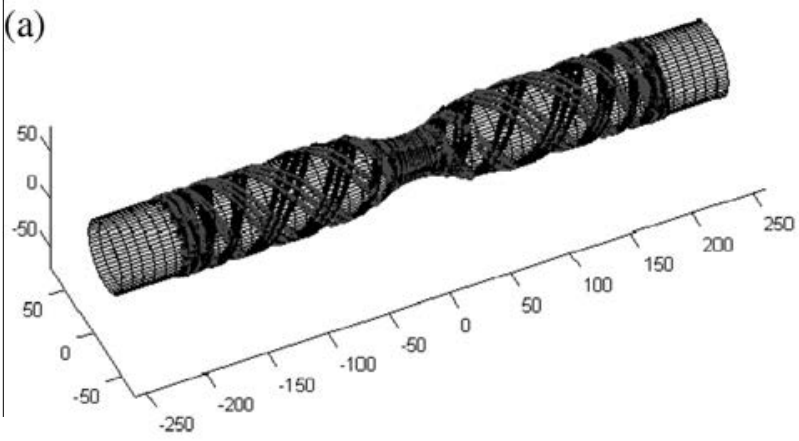

(c)

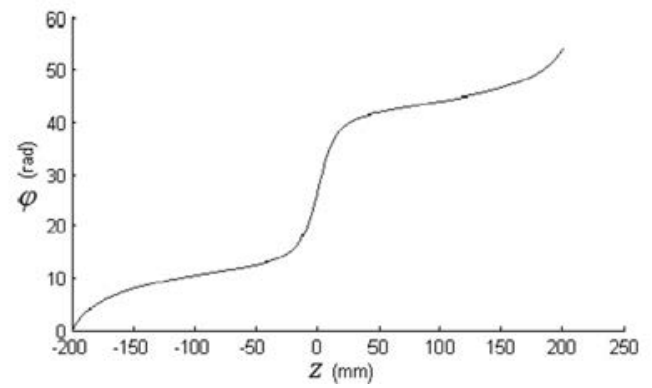

(b)

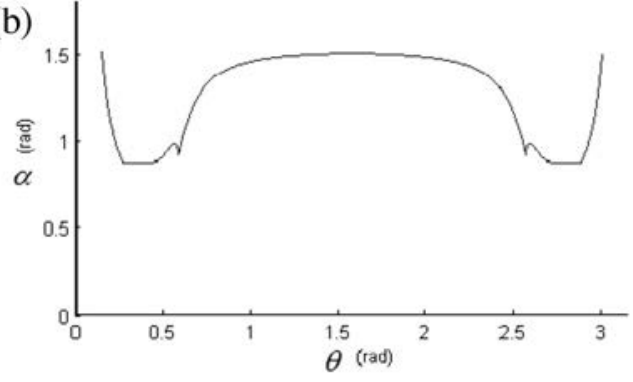

(d)

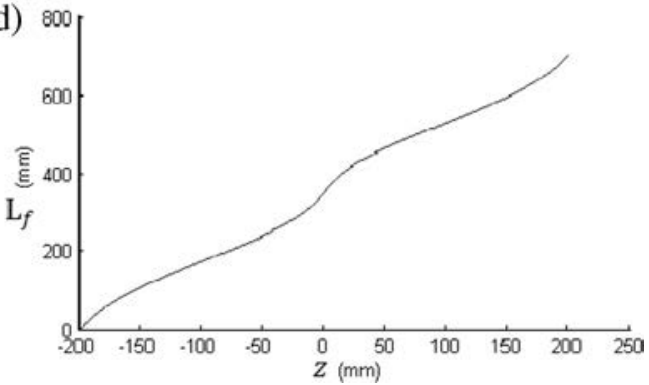

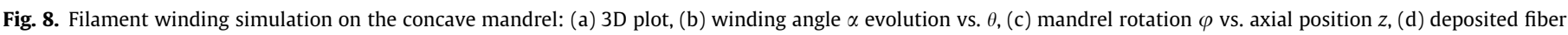
length $L_{f}$ vs. axial position $z$.

tion of the axisymmetric geometry is $w_{t} / \cos (\alpha)$; then by dividing it into the circumference the number of circuits $N_{p}$ required to fully cover is given by (28).

$N_{p}=\frac{2 \pi R}{w_{t}} \cos (\alpha)$

The number of circuits must be an integer number, otherwise there will be overlapping of the band edges creating unevenness of the outer surface of the winding when the value is rounded up; or gaps that will tend to fill up with resin and may result in decreased final fiber volume fractions on the opposite case [24]. These undesired defects can be eliminated by adjusting the actual winding angle or by changing the geometrical parameters involved such as $R$ or $w_{t}[8,24]$; or by introducing a controlled overlap to each subsequent circuit of the fiber $[20,21]$. In order to control the interweaving pattern, expression (29) allows to calculate the angle of rotation of the mandrel when the translating carriage reaches its end stroke at a dwell phase.

$2\left(\Omega+\varphi_{0}\right)(\bmod 2 \pi)=\frac{2 \pi N}{N_{p}}$

The term to the left represents the full turning angle expressed as a function of the total angle of one single circuit, $\Omega$; and the turning angle during the dwell phase, $\varphi_{0}$; the coefficient 2 comprises a full circuit. The term to the right represents the angular displacement in terms of fractions of the circumference counted from a departing position towards the next possible position by multiplying the angular dimension that corresponds to the width of the fiber along the hoop direction, $2 \pi / N_{p}, N$ times. The value of the band pattern $N$ allows to control the interweaving degree of the pattern and as previously mentioned, it must be mutually relatively with $N_{p}$, and $N<N_{p}$. The modulus of congruence 2 stands for complete turns by which the full turning angle $2\left(\Omega+\varphi_{0}\right)$ is divided to obtain the remainder $2 \pi N / N_{p}$.

To summarize, the procedure to obtain the turning angle at the dwell phase $\varphi_{0}$ implies to calculate $N_{p}$, to correct it so $N_{p} \in \mathbb{Z}$, to evaluate $N$, and finally to use the expression (29). The term $\Omega$ can be ideally estimated considering a fiber path with a fixed filament winding angle $\alpha$ from extreme to extreme (with no transition lead before the dwell phase), so [23],

$\Omega=L_{m} \tan (\alpha) / R$

\section{Fabrication procedure}

As previously stated, the trajectory all along the longitudinal axis $z$ of the mandrel is numerically known but not validated. To do so, two detachable mandrels considering the geometries under study were fabricated so as to demonstrate the feasibility of the mathematical procedure, as well as to pursuit with mechanical tests later on.

The mandrel consists of two hollow steel cylinders joined with pins at each side of a central detachable part, either the convex or the concave geometry. The convex geometry was machined using six different blocks stacked together and contour-turned using a hydraulic lathe tracer and a template defining the contour calculated by (19). During the machining process these blocks are supported along the radial direction with a hexagonal-section hollow bar, and fixed with pins (see Fig. 9). This support works as an attaching interface with the previously referred cylinders. Once the reinforcement is wound and polymerized, the mandrel is extracted piece by piece, starting from any of the steel cylinders, then the hexagonal-section hollow bar, and finally the bars forming the convex geometry. The concave geometry is simpler and it was contour-turned using two hollow steel bars, following the contour defined by (20). The full length of both mandrels is approximately $500 \mathrm{~mm}$, the length of both the convex and the concave geometry is $90 \mathrm{~mm}$. The radius of the cylindrical parts is $30 \mathrm{~mm}$.

Surface preparation is achieved by putting a high temperature Teflon adhesive tape at every gap so the water-based release agent Moulex 6000BG from Additek ${ }^{\circledR}$ applied over the entire metallic surface does not corrode the mandrel interior. Hot air is applied while the mandrel turns slowly once mounted and fixed on the winding machine. Three layers of the release agent are recom- 

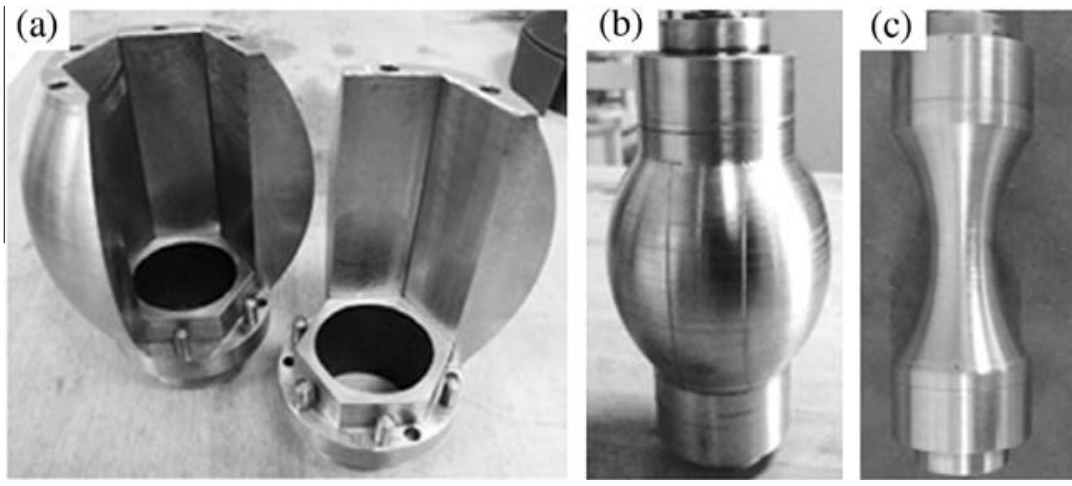

Fig. 9. Detachable mandrels: (a) convex part before assembly, (b) and after, (c) concave part.

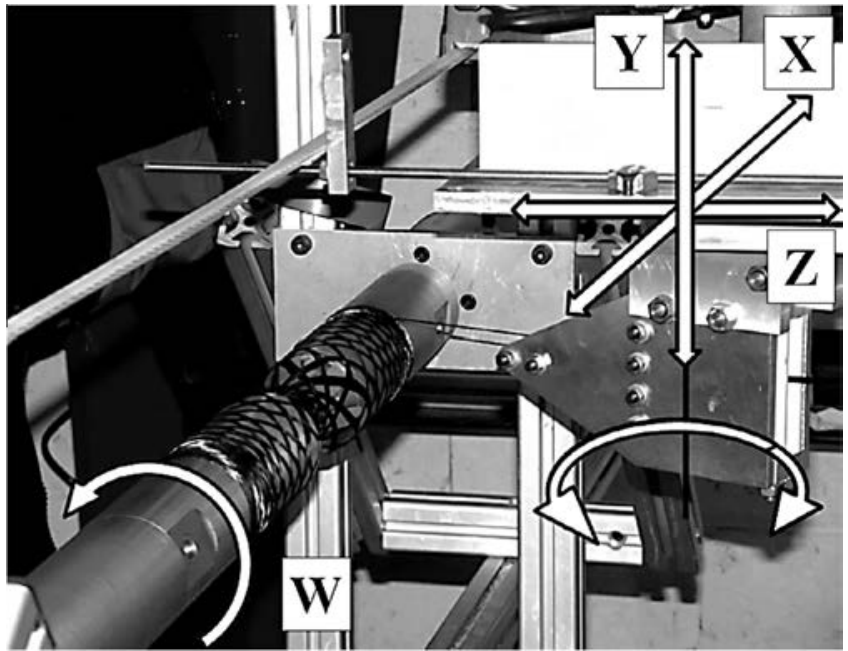

Fig. 10. Filament winding machine with concave mandrel.

mended as minimum, turning the surface from bright metal (metallic cylinders have a roughness about $3.2 \mathrm{Ra}$ ) to a whitish smooth coated surface.

The machine used for the filament winding is shown in Fig. 10; it is a Cartesian robot with three linear axes which are perpendicularly oriented at each other, with a free rotating delivery head and a rotating axis with a maximum angular speed of $3900 \mathrm{rpm}$ coupled to speed reducer (1:40), therefore the maximum winding speed is $97.5 \mathrm{rpm}$. It is computer driven using a Galil ${ }^{\circledR}$ PCI 1842 controller card. The reason for the selection of this type of machine and its programming software is due to its manufacturing flexibility, and programmable automation.

Fiber tension is applied with an unwind tension compensator model 800C012 from CTC Inc. The delivery point consists of a series of static rollers made of Teflon, disposed in a vertical and then in an horizontal position, considering the path of the fiber coming from the tension compensator; the said delivery head rest at the same height with respect to the upmost generatrix of the cylindrical parts of the mandrel. During filament winding, a small horizontal slippage of the fiber persists within the delivery rollers.

Reinforcement material is a $4 \mathrm{~mm}$ wide, unidirectional, highmodulus-carbon Prepreg tow, with identification number T700GC-12K-31E, and with an UF3369-100 resin system (resin content 29.5-30\%; Young modulus, 3.1 GPa; tensile strength, 92.4 MPa; elongation at brake $3.6 \%$, suitable for high-pressure cylinders) from TCR. According to manufacturer, three alternative curing cycles are suitable: (1) $4 \mathrm{~h} @ 121^{\circ} \mathrm{C}$; (2) $6 \mathrm{~h} @ 110^{\circ} \mathrm{C}$; and (3) $241 \mathrm{~h} @ 99^{\circ} \mathrm{C}$. Second option was chosen using a Nabertherm ${ }^{\circledR}$ oven with good results.
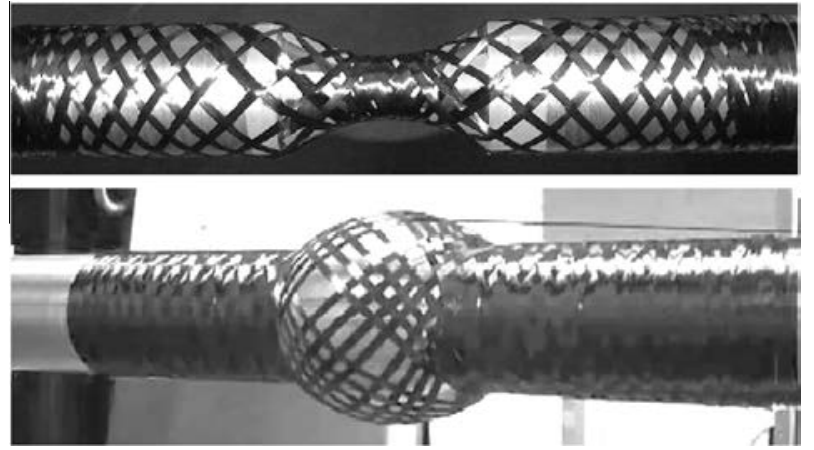

Fig. 11. Filament-wound convex and concave geometry.

Before starting any filament winding, the delivery head is positioned to a machine reference position. A simple device was adapted to measure the displacement along the axis parallel to the rotating axis of the mandrel, allowing to register the departing point for each filament winding.

Fig. 11 shows the results obtained for both geometries under study. During the fabrication of the convex mandrel no undesired slipping of the fiber was observed; the placement of the fiber is as expected. For concave mandrel, though the simulated process proposes geodesic trajectories, i.e. no sliding expected, this winding requires the operator intervention to ensure the path to be stable at the transition point; still a symmetrical winding pattern is obtained.

Demoulding of both mandrels was assisted using an Adamel Lhomargy ${ }^{\circledR}$ MTS DY25 tensile test machine (500 daN). The manual displacement feature was used to facilitate extraction of the cylinder attached to the crosshead. Then either the convex, or the concave central assembly were extracted piece by piece as previously explained and may be reused to produce a convex or concave composite structure again.

\section{Discussion and analysis}

In Fig. 6 the distribution of the filament winding angle as a function of $\theta$ evolves as expected: from a value close to $\pi / 2(1.57 \mathrm{rad})$ to an input value of $55^{\circ}(0.95 \mathrm{rad})$. The evolution of the filament winding angle is relatively fast and then it rests constant, confirming the loxodrome traced over the surface of the cylindrical mandrel. In Fig. 7 the same evolution is verified on each side of the mandrel. Once the filament winding angle reaches the same input value, it rests constant up to the value of $\theta$ that corresponds to the transition between the cylinder and the convex geometry, then it reduces slowly up to a value of $35^{\circ}$ ( $0.60 \mathrm{rad}$ ) approximately just 
at the half-length of the mandrel. In Fig. 8 (concave mandrel) the evolution of the filament winding angle is the opposite to that of the convex mandrel: once the angle reaches the input value of $55^{\circ}$ it increases slightly, then it reduces abruptly, and finally it increases rapidly until it reaches a value smaller to $\pi / 2$. This discontinuity is almost observed on the $\alpha-\theta$ graph, the other graphs, $\varphi-z$, and $L_{f}-z$ do not show any singularity or an important discontinuity. As previously stated, the concave mandrel requires the intervention of the operator at the transition point of the cylinder and the concave geometry, i.e. the point of the singularity observed on the $\alpha-\theta$ graph. It is necessary to control the filament winding evolution so a soft transition is achieved, and the increment of $\alpha$ may not be as important as in this case.

Being the friction coefficient a fundamental parameter to construct non-geodesic paths, attention should be put when considering its value. As previously referred, and according to [6], $\mu$ can vary between 0.2 and 0.4 . Wang et al. [18] considered a series of experiments to evaluate the influence of fiber band width, winding speed, roving tension, viscosity and two common filament winding materials: carbon and glass. For this analysis a value of 0.25 was considered, nevertheless according to their results, the slippage coefficient could be set at $0.32-0.34$ for most of the cases, so it would be recommended to consider a value comprised within this rank.

The Cartesian filament winding machine used in this analysis proved to place the fibers as desired, achieving a symmetrical and balanced pattern on both sides of the mandrel, despite the inertial lag that could arise from the carriage that supports the delivery head that is mounted at the end of the vertical axis, though measurements of the filament winding angle as calculated are desirable. Another possible source of error is the slippage of the fiber at the delivery point, so instead of using cylindrical rollers, it would be recommended to use grooved rollers, or to change the delivery head to a delivery eye.

\section{Conclusions}

A mathematical model for the determination of non-geodesic winding trajectories is presented, and validated via the fabrication of two axisymmetric generic geometries, one convex and the other concave, aiming to contribute to development of complex filament-wound structures as part of an integral design approach. The numerical tool expressly developed solves the general path equation and will serve as basis for further mechanical behavior models by integrating onto them filament winding parameters. As an alternative, the complex geometry but also the mechanical loading could be introduced as input data in order to define the slipping tendency according to $\theta$ parameter which optimizes the structure strength and the stacking sequence simultaneously - to be outlined in future publication.

For each geometry under analysis, a detachable metallic mandrel was designed and fabricated. Even though non-geodesic trajectories are designed, slippage of the fiber was observed, so further considerations related to static friction coefficient measurements should be considered. It is particularly necessary to integrate a friction function allowing a more precise control of fiber placement. Moreover, demoulding of the composite material C-Ep previously cured proved to be a technological challenge that implied the development of detachable, metallic mandrels. Other solutions were considered, nevertheless the state of the art, or their technological maturity proved to be insufficient. It can be stated that the maturity of filament winding models is closely related to the technological development of mandrels solutions.

\section{Acknowledgments}

The authors gratefully acknowledge the National Council of Science and Technology of Mexico (CONACYT, Consejo Nacional de Ciencia y Tecnología) for the doctoral scholarship of Erik Vargas Rojas, as well as the financial support given to him by the Franche-Comté Conseil Regional and the Université de Franche-Comté as part of the Doctorant Conseil program. Vargas also thanks the scientific advice from Mr. F. Gasquez.

\section{References}

[1] Dossier [online], Institut National de l'Environnement Industriel et des Risques (France), Dossiers thématiques/Dossier Hydrogène. Maîtriser le risque pour un développement durable, l'hydrogène, vecteur d'énergie. May 2010. Available from World Wide Web: <http://www.ineris.fr/centredoc/dossier-hydrog\% C3\%A8ne-ineris-def.pdf>.

[2] Farines L, Thiebaud F, Perreux D. Form-fitted tank. In: ECCM15 - 15th European conference on composite materials. Venice, Italy; 24th-28th June, 2012.

[3] Anderson A, Bowman K, Teufel P. One piece closed-shape structure and method of forming same. US Patent No. US7431239 B2; October, 2008.

[4] Anderson A, Allman M, Ashton L, Simpson C, Ta'ala B, Whute T. Filament winding apparatus and methods of filament winding filament, US Patent No. 7124797 B2; October, 2006.

[5] De Carvalho J, Lossie M, Vandepitte D, Van Brussel H. Optimization of filamentwound parts based on non-geodesic winding. Compos Manuf 1995;6(2): $79-84$.

[6] Koussios S, Bergsma OK, Beukers A. Filament winding. Part 1: determination of the wound body related parameters. Compos: Part A 2004;35:181-95.

[7] $\mathrm{Zu} \mathrm{L}$, Koussios S, Beukers A. Design of filament-wound isotensoid pressure vessels with unequal polar openings. Compos Struct 2009;92:2307-13.

[8] Gasquez F. Étude des réservoirs entièrement bobinés en composites destinés au stockage de l'hydrogène sous pression: cas des réservoirs type III, Doctoral thesis, Université de Franche-Comté; 2008.

[9] Koussios S. Filament winding: a unified approach [Doctoral thesis]. Technische Universiteit Delft; 2004.

[10] Lipschultz M. Schaum's outline of differential geometry. Europe: McGraw-Hill Education; 1969.

[11] Kreyszig E. Differential geometry. New York: Dover Publications; 1959.

[12] Scholliers J, Van Brussel H. Computer-integrated filament winding: computerintegrated design, robotic filament winding and robotic quality control. Compos Manuf 1994;5(1):15-23.

[13] Selected chapters of geometry and mathematics with mathematica [online], University of Zagreb, Faculty of Civil Engineering (Croatia). In: Gorjanc S, Hak $\mathrm{S}$, Uroš M, editors. Normal, Gaussian and mean curvatures at the regular point of a surface; 2004. Available from World Wide Web: <http://www.grad.hr/ itproject_math/Links/sonja/gausseng/introduction/introduction.html>.

[14] Weisstein W. Surface of revolution from MathWorld, a Wolfram Web Resource. <http://mathworld.wolfram.com/SurfaceofRevolution.html>.

[15] Bulletin [online], Société Mathématique de France. Barré. Théorie générale des surfaces engendrées par une hélice circulaire, Tome 1973; 41: 242-39, Available from World Wide Web: <http://www.numdam.org/item?id=BSMF_ 1913_41_242_1>.

[16] Houcque D., Applications of Matlab: ordinary differential equations; 2005. Available from World Wide Web: <http://www.math.unipd.it/ alvise/ CS_2008/ODE/MFILES/ode.pdf>.

[17] Chapra S. Numerical methods for engineers. 3rd ed. McGraw Hill; 1998.

[18] Wang R, Weichang J, Wenbo L, Yang F, He X. Slippage coefficient measurement for non-geodesic filament winding process. Compos: Part A 2011;42:303-9.

[19] Lazuardi D. Une approche du rôle des contraintes internes liées à l'élaboration sur le comportement des composites stratifiés [Doctoral thesis]. France: Université de Franche-Comté; 1988.

[20] Koussios S. Influence of the fiber bundle bandwidth on the determination of a winding pattern. In: Proceedings of the European conference on spacecraft structures, materials and mechanical testing; 2000. p. 375-81.

[21] Moreno H. Monitoring de la fabrication de tubes composites réalisés par enroulement filamentaire et comportement mécanique sous pression externe [Doctoral thesis]. Université Paul Sabatier; 2006.

[22] Moreno H, Douchin B, Collombet F, Davies P. Precise positioning of unit cells and embedded instrumentation for fabrication and pressure testing of filament wound tubes. In: 11th European conference on composite materials; 2004. p. 10.

[23] Rousseau J, Perreux D, Verdière N. The influence of winding patterns on the damage behavior of filament-wound pipes. Compos Sci Technol 1999;59:1439-49.

[24] Priestley AP. Programming techniques, computer-aided manufacturing, and simulation software. In: Peters ST, editor. Composite filament winding. ASM International; 2011. p. 35-47. 